\title{
Designing a Navigation System for Older Adults: A Case Study Under Real Road Condition
}

\author{
Perrine Ruer ${ }^{1(\bowtie)}$, Damien Brun ${ }^{2}$, Charles Gouin-Vallerand ${ }^{3}$, \\ and Évelyne F. Vallières ${ }^{4}$ \\ ${ }^{1}$ HEC Montreal, Montreal, QC H3T2A7, Canada \\ perrine.ruer@hec.ca \\ ${ }^{2}$ Université TÉLUQ, Montréal, QC H2S3L5, Canada \\ brund@acm.org \\ 3 École de Gestion, Université de Sherbrooke, Sherbrooke, QC J1K2R1, Canada \\ charles.gouin-vallerand@usherbrooke.ca \\ ${ }^{4}$ Centre LICEF, Université TÉLUQ, Montréal, QC H2S3L5, Canada \\ evelyne.vallieres@teluq.ca
}

\begin{abstract}
Recent research allows envisioning what kind of sensory devices could be used for drivers' navigation in the future, particularly for older adults. Population all around the world is aging, older adults will be more on the road. In this paper, we present a contact-less navigation system dedicated to this category of people based on a mobile head-up display. The aim of this system is to preserve their mobility in order to promote their autonomy and daily social activities. To do so, we interviewed older drivers to design a driving assistance system and we assessed the system under real road conditions $(\mathrm{N}=34)$ with measurement of older drivers' mental workload and the adequacy of users' expectations. We emphasize the need to combine both measures of mental workload. The contribution aims at providing a richer understanding of how older people experience navigation technologies and to discuss the design recommendations of digital devices for older people.
\end{abstract}

Keywords: Older people $\cdot$ Driving assistance system $\cdot$ Mental workload $\cdot$ Field studies

\section{Introduction}

Some research in Human-Computer Interaction (HCI) focuses on the kind of sensory devices that could be used for a driver's navigation in the future. That is convenient for the case of older drivers. This category of drivers will be more and more on the road in the next decades as shown from demographics prediction. Many physical and cognitive changes are associated with the aging process and could have negative impacts on driving activity. It is essential to preserve their mobility in order to continue to promote their autonomy and to have daily social activities.

In the last decade, little focus has been given to the driving assistance system focusing on older drivers' mobility that could help them to drive safely in the long 
term. To promote the autonomy of older drivers, one suggestion is to design navigation systems for and with the concerned users [1]. Several new cars are equipped with different driving assistance systems that have the potential to help older people to drive more safely as long as they are accepted and perceived as useful by these users with special needs and characteristics. Therefore, the aim of the present research was to propose a driving assistance system to promote driving for older drivers considering their aging impairments.

Usability is something to consider when dealing with older adults. Older people do not have the same familiarity with new technologies that young people [2]. If older drivers question the system, this may cause them to stop using the system. It is necessary to understand their expectations of technologies. In addition, if people are familiar with a system, they are likely to have a more favorable opinion of it [3].

After the definition of needs and expectations with older drivers, we designed a driving assistance system corresponding as closely as possible with these ones. The study involves the assessment of the newly designed driving assistance system from real-world experiments with 34 participants measures of objective and subjective mental workload. Our contributions are (1) to design a driving system assistance for older drivers based on Head-Up Display interactions (2) to assess the value of the system with the assessment of different measures of mental workload.

\section{Related Works}

The development of transportation technologies makes possible to maintain older drivers' mobility by allowing them to use a personal vehicle to reach their needs. Invehicle systems support drivers. Their aims are to increase safety and save lives. These systems can help drivers in critical situations or in unfamiliar environments by giving driving information. For example: turn on your left at the next intersection [4]. However, these systems are developed most of the time for all drivers and few of them are assessed with older drivers [4]. Especially since older drivers have their own usability problem with computing systems [2]. They have particular needs and expectations which could differ from other categories of people (for instance, young people) [5].

A bad conception of a driving assistance system leads to the perception of the complexity of the driving task and a higher risk of accidents [6]. Furthermore, a bad conception induces more inconvenience for older drivers, whereas the system's role is to encourage mobility and security of the driver who uses it [6, 7]. User tests allow to assess a system and to verify its credibility [8]. A previous study was made with older drivers to assess a new driving system during user tests. Results indicated the system was positively perceived. Participants were able to better understand the benefits and limits of it [9].

Head-Up Display (HUD) is a recent interesting driving assistance system for older drivers. It is defined as a display mode which is used to present different information to the driver's field view (speed information, warnings or other indicators) [10]. The benefits of HUD are to allow quick reaction time; earlier detection of road hinders or to let more time to scan the traffic scene [10-12]. Most research focuses on the visual 
canal to provide information during the driving task. Older drivers are more sensitive to visual impairments with aging. Several authors agree that the main beneficiaries of HUD systems are aging people because of their narrower field of vision [11]. HUD is used to limit off-field sightings of the road scene by transmitting information directly into the driver's field of view and allowing a controlled driving task [12, 13]. A study indicated that the use of a head-up display system was assessed by participants as it was easy to use [10]. HUD has great potential to improve comfort for older drivers.

Interaction with driving assistance system can increase task complexity having consequences regarding safety (i.e. distraction or increased fatigue) [13]. Mental workload is a good indicator of distraction during the evaluation of a new driving assistance system [14]. A system should not increase distraction involving a higher mental workload with too much information to process [4, 13]. Mental workload allows assessing if a computing system supports or increases people's cognitive resources needed, particularly when a driving assistance system is added. The system could either assist the drivers or disturb them to drive more safely, especially older drivers $[1,4,6]$. Indeed, older drivers are more sensitive to distraction during dual tasks due to the aging process [15]. Mental workload is not directly observed. Two main measures are generally used to do so: an objective measure and a subjective measure. Objective measure is physiological cues such as heart rate, pupillary diameter, skin temperature, electrodermal activity $[16,17]$. To detect these clues, biometric sensors are used to measure the body's reactions reflecting the driver's mental workload [17]. Subjective measures are questionnaires or scales to assess a specific event such as NASA Task Load Index (NASA-TLX). It is a multidimensional assessment tool with six different dimensions: mental demand, physical demand, temporal demand, effort, performance, and frustration [18]. It is strongly recommended to combine these different type of measurements in order to better assess mental workload when using a driving assistance system [14].

During the development of a driving assistance system, it is recommended to offer user tests, particularly for older drivers. The measurement of mental workload makes it possible to predict the influence (positive or negative) of a new driving assistance system during a driving task. The HUD seems to be an interesting system for older drivers, especially with the reduction of their field of view.

\section{Methodology}

The study consists of an implementation phase (3.1) and an exploratory study under real conditions (3.2).

\subsection{Implementation Phase}

Driving Assistance System Choice. Among driving assistance systems, HUD is interesting for older drivers. In our study, we used a commercialized system named HudwayGlass. This system consists of a phone holder and a tinted plastic blade covered with a mirror coating to reflect phone screen. This system can easily be purchased 
by any driver from the company's website ${ }^{1}$. The HudwayGlass HUD has three advantages: (1) the system is universal and can be used in all vehicles, (2) with any smartphone and (3) is customizable, letting the possibility to design any kind of mobile application adapted for this type of display (Fig. 1).

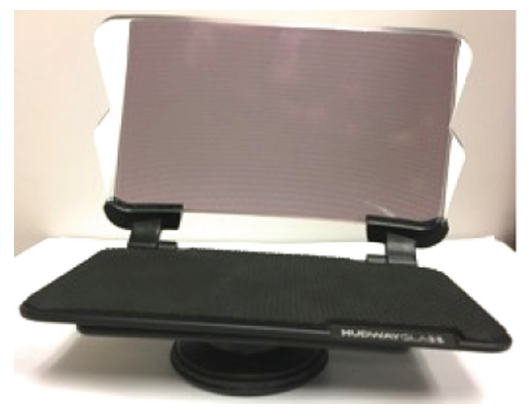

Fig. 1. HudwayGlass HUD

The HudwayGlass company offers specific mobile applications, but for our research, we developed our own mobile application for it to be well tailored for our intended older drivers.

Design of Mobile Applications. Regarding the design process, we assessed expectations of older drivers relative to mobile application interfaces with interviews. In these, we asked participants about pictogram and color for three types of interfaces. We implemented the most preferred interfaces in a mobile application (app.) named "ARDriving Assistant". The mobile app has been divided into two sub-app. The first one presents information to the driver. This sub-app was built with the Unity software and was configured to run on an Android phone. It projects the information to the driver through the HUD interface. The objective was to provide driving notifications with pictograms and colors. These choices are important when designing technology interfaces [19]. Figure 2 presents the three selected interfaces according to expectations collected.
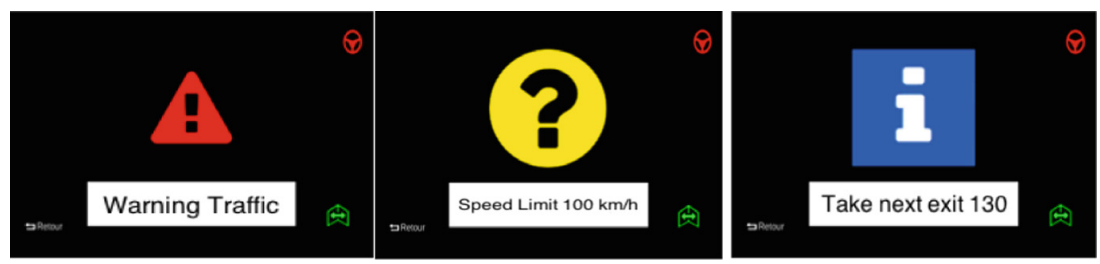

Fig. 2. Interfaces from the first sub-app. (in order of presentation: alert, information and advice)

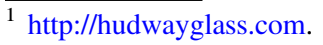


The second sub-app allows control of notifications sent to the driver and collects data. The objective was to control the first sub-application by collecting data during driving for later analysis (i.e. speed, breaking, position, etc.). The application consists of a scrolling view containing several buttons including a dialog box to configure and start sensor monitoring (Fig. 3).

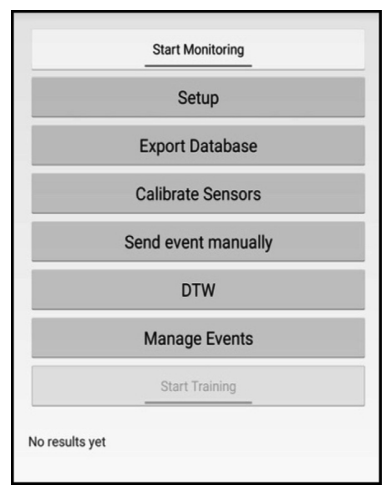

Fig. 3. Interface from the second sub-app.

This application was developed with the Android Studio software and the application code is available on GitHub (https://github.com/limvi-licef/AR-driving-assistant).

\subsection{Assessment Under Real-World Conditions}

The success or failure of a driving assistance system lies in a design for the targeted drivers.

Participants. Thirty-nine (39) participants were recruited, but only 34 took part in this study ( $41 \%$ of whom were women). Five participants withdrew a few days before their appointment for various reasons (loss of interest in the study, a problem with their personal vehicle or health problem). The sample was composed of older people between 60 and 85 years old (mean age: 68.8 years, standard deviation: 5.91). Participants lived in Montreal area (Quebec, Canada). We recruited participants with a local paper ad and with the university e-journal. Each participant was reached by phone to answer a pre-recruitment questionnaire to verify that they matched with the selection criteria. These criteria were to be 60 years old or more, to hold a valid driving license, to drive each week on a regular basis and to be in good health. To confirm the relevance of our system, we compared several measures by dividing our sample. Two groups were randomly composed: a control group and an experimental group. The experimental group $(\mathrm{N}=17)$ drove with the prototype system, while the control group $(\mathrm{N}=17$ ) did not have the prototype system during driving. The control group was used as a reference group for the comparison of the collected measures. They were instructed to perform a routine driving task. 
Procedure. Participants were invited to read and fill out a consent form. Once completed, they answered a questionnaire to collect sociodemographic variables, such as age, gender or driving experience. Participants were asked to drive on a highway in the greater Montreal area. The journey distance was a loop of $63 \mathrm{~km}$ and was completed in $40 \mathrm{~min}$. All participants must respect traffic laws and driving speed limitations. The route drivers needed to drive was to follow the highway until exit 160 . Then, they made a turn back to the outset. The journey was selected to a limited amount of traffic on the highway, with fewer stimuli than in urban road and to have a reproducibility of the scenario during experiments. Highway driving is known not to increase mental workload, unlike an urban road with the presence of less disruptive elements (e.g. pedestrians, parked vehicles, etc.) [14]. We also avoided as much as possible peak hours (8:00 am-9:00 am and 4:00 pm-5:00 pm) and intense traffic. We chose experiments in real-world conditions because they allow assessing variables with dynamic factors coming from the driving context [18]. Finally, they answered a questionnaire about their overall experience and received 30 Canadian dollars for their participation.

Collected Measures. We collected two objective measures: skin temperature and electrodermal activity. These two measures allow the assessment of the driver's mental workload [14, 17, 20]. During the driving task, all participants wore two biometric sensors from the Captiv' Solutions. These sensors are affixed with self-gripping tape and can be positioned at different locations on the body such as wrists or elbows. Each sensor weights 20 grams, measures $52 \mathrm{~mm} \times 25 \mathrm{~mm} \times 14 \mathrm{~mm}$. Their sampling frequency is $32 \mathrm{~Hz}$ with a resolution of 16 bits and $0.05{ }^{\circ} \mathrm{C}$. The amount of data collected is 1920 per minute for each sensor. These sensors are conveniently connected to a wireless box. Software is available to synchronize and display the data in real time, then record the data collected and perform subsequent analyses. Sensors were positioned on participants' left-hand fingers and a medical tape was added to hang the wires to the hand. They cause slight discomfort but do not prevent the steering wheel from being grasped or the execution of driving gestures with the right hand [21].

Regarding the subjective measure NASA-TLX, Participants answered it in the car straight after the driving task on the highway and before they got out. The first step of the NASA-TLX was to assess the six dimensions (mental demand, physical demand, time demand, effort, performance, and frustration) on scales ranging from low to high. We presented a definition for each dimension to ensure the participants' understanding. Then, participants carried out a comparison phase and chose from two dimensions that the experimenter said out loud. For example: "indicate the dimension required the most effort while driving between mental demand or performance".

All participants drove the University instrumented vehicle, named LiSA car. They all drove this vehicle to have better comparison and not have a vehicle change that would potentially influence the data collection. This vehicle is a Nissan Versa 2007 equipped with a data logger (AIM Evo4) and a computer embedded on board. An experimenter was placed in the back seat of the vehicle. The experimenter indicated to the participant that he or she could not speak during the whole driving task to not influence the driver. The experimenter could track the collected data in real time and complete the task with a paper-pencil statement with driving information (i.e. traffic, 
participant comments). The experimenter sent one of the three interfaces with the second sub-application to the first sub-application.

\section{Results}

As a reminder, our objective is to propose a driving assistance system to promote safe driving for older drivers. We did assessments under real-road conditions in order to confirm if this system meets the expectations of older drivers. First, we present results from the objective mental workload data (skin temperature and electrodermal activity) for control and experimental groups. Then we present the subjective mental workload's results for both groups. We study comparison between objective and subjective mental workload. Finally, we describe system usability assessment results completed by the experimental group.

\subsection{Objective Mental Workload}

Control group participants had a lower average skin temperature than experimental group participants $\left(31.34\right.$ Celsius degree $\left({ }^{\circ} \mathrm{C}\right)$ vs $\left.31.81{ }^{\circ} \mathrm{C}\right)$. There is a small gap between the two groups for the skin temperature. For electrodermal activity, mean is lower for the control group than for the experimental group (2.20 micro-Siemens $(\mu \mathrm{S})$ vs $3.51 \mu \mathrm{S}$ ). There is a higher variability into the experimental group. Table 1 shows descriptive results for each sensor by group.

Table 1. Descriptive results.

\begin{tabular}{l|l|l|l}
\hline & & \multicolumn{3}{|l}{ Group } \\
\cline { 3 - 4 } & & Control group & Experimental Group \\
\hline \multirow{2}{*}{ Skin temperature } & Mean & $31.34{ }^{\circ} \mathrm{C}$ & $31.81^{\circ} \mathrm{C}$ \\
\cline { 2 - 4 } & $\mathrm{Sd}$ & $2.3{ }^{\circ} \mathrm{C}$ & $2.02{ }^{\circ} \mathrm{C}$ \\
\hline \multirow{2}{*}{ Electrodermal activity } & Mean & $2.20 \mu \mathrm{S}$ & $3.51 \mu \mathrm{S}$ \\
\cline { 2 - 4 } & $\mathrm{Sd}$ & $1.59 \mu \mathrm{S}$ & $3.29 \mu \mathrm{S}$ \\
\hline
\end{tabular}

A Mann-Whitney U test was used to compare the group's influence on skin temperature and electrodermal activity. There are no significative group's differences neither for skin temperature ( $\mathrm{U}: 136.50 ; \mathrm{p}=.78 ; \mathrm{n}=34$ ) nor for electrodermal activity (U: $124 ; \mathrm{p}=.50 ; \mathrm{n}=34$ ).

Even if participants in the experimental group show higher skin temperature and electrodermal activity than participants in the control group, the differences are nonsignificant. Additional experiments should confirm whether there is a difference which can be inferred by the context or the stress induced by experimenting a new driving assistance system. 


\subsection{Subjective Mental Workload}

For the control group, the most relevant dimension was mental demand (mean: 104.6), for the experimental group, it was performance (mean: 152.94). Figure 4 presents descriptive results.

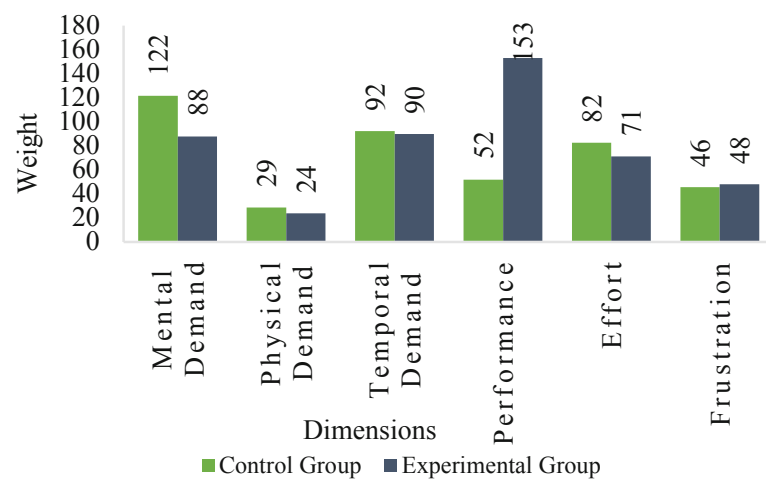

Fig. 4. NASA-TLX descriptive results.

A Mann-Whitney U test was used to compare groups' assessment of NASA-TLX dimensions. The hypothesis was that participants, regardless of group, would answer in the same way. This hypothesis was confirmed for five dimensions: mental demand (U: 121.5; $\mathrm{p}=.43 ; \mathrm{n}=17$ ), physical demand ( $\mathrm{U}: 124 ; \mathrm{p}=.49 ; \mathrm{n}=17)$, temporal demand (U: $134.5 ; \mathrm{p}=.73 ; \mathrm{n}=17$ ), effort (U:143.5; $\mathrm{p}=.97 ; \mathrm{n}=17$ ) and frustration (U:125; $\mathrm{p}=.52 ; \mathrm{n}=17)$. The hypothesis was rejected for performance. There is a significant difference for the performance dimension between control and experimental groups (U: $56 ; \mathrm{p}<.005 ; \mathrm{n}=17$ ). Subjective perception of participants mental workload is higher for performance. This result suggests that the system (HUD and driving mobile app.) had an impact on the performance dimension of driving, but not on the other dimensions of the NASA-TLX. It was more demanding for participants who drove with the system than those who drove without it.

\subsection{Comparison of Objective and Subjective Mental Workload}

Following the results presented above, a comparison was made on the three measures of mental workload (objective and subjective). We wanted to identify an effect between means of skin temperature; mean electrodermal activity; subjective conditions: mean total weight of NASA-TLX dimensions. The inter-subject factor was the group condition (control or experimental). Table 2 indicates no significant effect of objective and subjective mental workload conditions according to the group $(\mathrm{F}[30]=.954, \mathrm{p}<.50)$. 
Table 2. Multivariate ANOVA test.

\begin{tabular}{l|l|l|l|l|l|l}
\hline Test name & Value & $\mathrm{F}$ & Hypoth. Df & Sig. of F & Sum of square & $\mathrm{p}$ \\
\hline Pillais & .09 & .95 & 3.00 & .47 & .09 & .24 \\
\hline Wilks & .91 & .95 & 3.00 & .43 & .09 & .24 \\
\hline Hotelings & .09 & .95 & 3.00 & .43 & .09 & .24 \\
\hline Roys & .09 & .95 & 3.00 & .43 & .09 & .24 \\
\hline
\end{tabular}

This result indicates that both groups did not differ on the measures of objective and subjective mental workload. It appears the system (HUD and driving application) is an interesting modality for a driving assistance system for older drivers.

\subsection{System Usability Assessment}

After the driving task, participants from the experimental group $(\mathrm{N}=17)$ were asked to answer a questionnaire for assessing the system usability. Measures assessed were the satisfaction of the driving experiment, the strengths and the weaknesses of the system, and to judge the system usefulness. The overall driving experiment was rated satisfactory or very satisfactory for $94 \%$ of participants. Participants were surveyed about the strengths and weaknesses of the system (i.e. HUD and interfaces). They had to rate five strengths: reliable, high-quality, useful, singular and ergonomic. Classified in order from best to least strength, the first was its ergonomics, its usefulness, its singularity, its high-quality rate, and the last its reliability. The five weaknesses were: seems too expensive, impractical, ineffective, poor quality and unreliable. Amongst these, the first is its poor quality, its unreliability, its inefficiency, its impracticality, and it seems too expensive. These strengths and weaknesses allow us to determine what was considered interested and what has to be improved.

Most participants indicated that the system did not interfere during the driving task (70\%). It means that the system in the participants' field of view is not perceived as a visual discomfort. About usefulness, $47 \%$ of participants considering the system useful or somewhat useful compared to $53 \%$ who considered it useless or rather useless. Subsequently, a question was asked about the benefit of the system to help driving safely. The system was rated as very beneficial or beneficial for $41 \%$ of participants. After testing the system, a quarter of participants would be interested in purchasing the system (24\%). And the system did not seem like other computer systems with which participants were familiar $(58 \%)$. The system was considered different from other known computing systems. These different aspects assessed allow us to know which ones need to be improved or preserved.

\section{Discussion - Conclusion}

Driving assistance systems are a solution to support the older drivers in their task. However, this type of assistance must be adapted and designed to meet the real needs of drivers. The assessment of the system influenced the subjective driving performance 
of older drivers, but not the objective mental workload where results did not indicate a significant difference between the two groups. Results of this work confirm the usefulness of measuring the mental workload of older drivers objectively and subjectively. Results of objective mental workload suggested there is no increased for the experimental group who assessed the system. Comparing both groups (control and experimental), mean skin temperature and mean electrodermal activity was slightly higher for drivers who had to assess the system. It means that the system tested would cause a slight increase in mental workload during driving activity, although there is no significant difference between groups. Objective mental workload measurements do not always correspond to the participants' feelings. It is the reason that we added to our objective measurements a subjective measure (NASA-TLX scale). This combination of mental workload measures should be encouraged in future research of older drivers. Our results indicated only one significant difference between the experimental and control groups: the performance dimension. The experimental group appears to have found it more difficult to drive than the control group. One explanation may be due to the novelty of the task, rather than the fact of driving with the system per se. Experimenting with a new system may have caused more anxiety. Combining mental workload measures is therefore recommended [14].

This study proposed to develop and assess new material based on expectations of older drivers in Quebec province (Canada). When a driving assistance system is designed with the intended users, it meets the expectations of users and will tend not to increase mental workload. The whole study provides new knowledge to think about improving driving for older drivers. A practical contribution is a development of HUD with adapted mobile applications which can be deployed quickly on the market and offered to older people by insurance companies.

In this study, the time to assess the system is a limitation. Participants discovered the system and drove with it for less than one hour. Since familiarity encourages opinion and system use [3], a longitudinal study is a proposal for future research in order to assess the system several times to encourage its usefulness.

As we wanted to validate the different interfaces with older drivers, it depended on the driving habits of each driver. Some would not have had as much information from the mobile app. while driving as they received during the experiments. It is necessary to propose a new study with the presentation of interfaces according to the context of the driving task through the analysis of data in real time. In the current mobile app., it is possible to collect contextual data (speed, brakes, acceleration, localization, etc.). It might be interesting to also include a data mining technique (e.g. decision tree) for realtime analysis of driving data. Future research could follow this direction and offer a personalized interface to the driver based on the driving context in real time.

Acknowledgments. This work was supported by MITACS. We thank all the participants from the user study and staff who wrote and provided helpful comments. 


\section{References}

1. Reimer, B.: Driver assistance systems and the transition to automated vehicles: a path to increase older adult safety and mobility? Public Policy Aging Rep. 24(1), 27-31 (2014)

2. Fisk, A.D., et al.: Designing for Older Adults: Principles and Creative Human Factors Approaches. CRC Press, Washington, DC (2009)

3. Duncan, M., et al.: Enhanced mobility for aging populations using automated vehicles (2015)

4. Eby, D.W., Molnar, L.J.: Has the time come for older driver vehicle? J. Ergon. (2014)

5. Williams, D., et al.: Considerations in designing human-computer interfaces for elderly people. In: 2013 13th International Conference on Quality Software. IEEE (2013)

6. Simões, A., Pereira, M.: Older drivers and new in-vehicle technologies: adaptation and longterm effects. In: Kurosu, M. (ed.) HCD 2009. LNCS, vol. 5619, pp. 552-561. Springer, Heidelberg (2009). https://doi.org/10.1007/978-3-642-02806-9_64

7. Davidse, R.J.: Older drivers and ADAS: which systems improve road safety? IATSS Res. 30 (1), 6-20 (2006)

8. Castillejo, E., Almeida, A., López-de-Ipiña, D.: User, context and device modeling for adaptive user interface systems. In: Urzaiz, G., Ochoa, Sergio F., Bravo, J., Chen, L.L., Oliveira, J. (eds.) UCAmI 2013. LNCS, vol. 8276, pp. 94-101. Springer, Cham (2013). https://doi.org/10.1007/978-3-319-03176-7_13

9. Reimer, B., Mehler, B., Coughlin, J.F.: An evaluation of driver reactions to new vehicle parking assist technologies developed to reduce driver stress. New England University Transportation Center, Massachusetts Institute of Technology, Cambridge (2010)

10. Tretten, P., et al.: An on-road study of head-up display: preferred location and acceptance levels. In: Proceedings of the Human Factors and Ergonomics Society Annual Meeting. Sage Publications, Los Angeles (2011)

11. Pauzie, A.: Head up display in automotive: a new reality for the driver. In: Marcus, A. (ed.) DUXU 2015. LNCS, vol. 9188, pp. 505-516. Springer, Cham (2015). https://doi.org/10. 1007/978-3-319-20889-3_47

12. Tufano, D.R.: Automotive HUDs: the overlooked safety issues. Hum. Factors 39(2), 303311 (1997)

13. Kim, S., Dey, A.K.: Augmenting human senses to improve the user experience in cars: applying augmented reality and haptics approaches to reduce cognitive distances. Multimedia Tools Appl. 75, 1-21 (2015)

14. Schneegass, S., et al.: A data set of real world driving to assess driver workload. In: Proceedings of the 5th International Conference on Automotive User Interfaces and Interactive Vehicular Applications. ACM (2013)

15. Lemercier, C., Cellier, J.-M.: Les défauts de l'attention en conduite automobile: inattention, distraction et interférence. Le travail humain 71(3), 271-296 (2008)

16. Collet, C., Salvia, E., Petit-Boulanger, C.: Measuring workload with electrodermal activity during common braking actions. Ergonomics 57(6), 886-896 (2015)

17. Solovey, E.T., et al.: Classifying driver workload using physiological and driving performance data: two field studies. In: Proceedings of the SIGCHI Conference on Human Factors in Computing Systems. ACM (2014)

18. Hart, S.G.: Theory and measurement of human workload. Hum. Prod. Enhanc. 1, 396-456 (1986)

19. Leung, R., McGrenere, J., Graf, P.: Age-related differences in the initial usability of mobile device icons. Behav. Inf. Technol. 30(5), 629-642 (2011) 
20. Shi, Y., et al.: Galvanic Skin Response (GSR) as an index of cognitive workload. In: ACM CHI Conference Work-in-Progress (2007)

21. Clarion, A.: Electrodermal indices for mental workload analysis in car driving. Université Claude Bernard - Lyon I (2009)

Open Access This chapter is licensed under the terms of the Creative Commons Attribution 4.0 International License (http://creativecommons.org/licenses/by/4.0/), which permits use, sharing, adaptation, distribution and reproduction in any medium or format, as long as you give appropriate credit to the original author(s) and the source, provide a link to the Creative Commons license and indicate if changes were made.

The images or other third party material in this chapter are included in the chapter's Creative Commons license, unless indicated otherwise in a credit line to the material. If material is not included in the chapter's Creative Commons license and your intended use is not permitted by statutory regulation or exceeds the permitted use, you will need to obtain permission directly from the copyright holder.

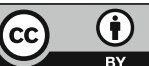

